\title{
Anti-cancer therapies affected by gut microbiota
}

T wo papers recently published in Science show that the gut microbiota can alter a patient's response to anti-cancer therapies.

The gut microbiota is known to affect immunity, metabolism and tissue development. However, little is known about whether and how the gut affects the response of patients to chemotherapy, leading two research teams to investigate this question.

In the first study, a team from France investigated the effects of cyclophosphamide on the microbiota in the small intestine, as well as how these effects influence the antitumour immune response. Sophie Viaud and co-workers characterized the inflammatory status of the gut epithelial barrier of mice $48 \mathrm{~h}$ after they had been given cyclophosphamide. The treatment resulted in shortening of the villi in the small intestine and discontinuities of the epithelial barrier; in addition, the number of goblet cells in the villi increased, as did the number of Paneth cells in intestinal crypts. In the duodenum of the treated mice, lysozyme (an antibacterial enzyme) expression was upregulated. At the same time, commensal bacteria were translocated to the mesenteric lymph nodes and spleen as a result of the damage caused to the gut epithelial barrier.

The overall composition of the gut microbiota was also analysed. Dysbiosis

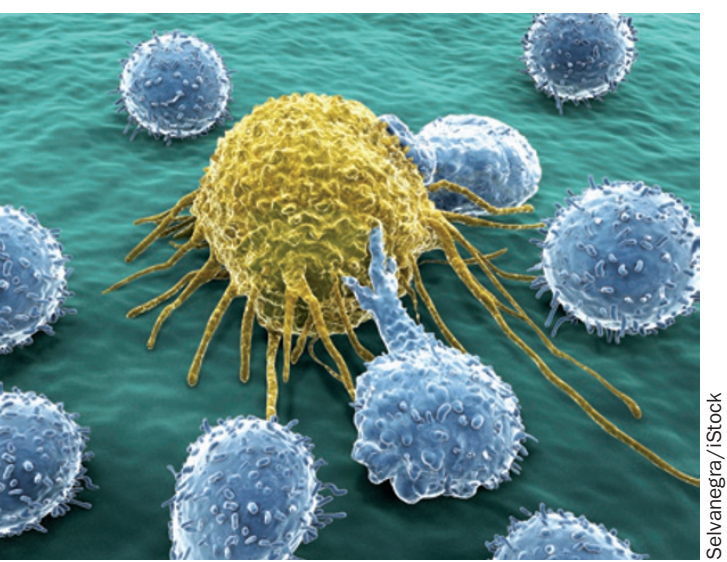

did not occur within $48 \mathrm{~h}$ of administering cyclophosphamide, but was detectable within 7 days. In particular, levels of Firmicutes bacteria and lactobacilli and enterococci in the small intestine were reduced after treatment.

The researchers also demonstrated that cyclophosphamide induced $\mathrm{CD} 4^{+}$ $\mathrm{T}$ cells in the spleen to become IL-17 producers, with the gut microbiota being indispensable for this process. Longterm treatment with broad-spectrum antibiotics was shown to limit the efficacy of cyclophosphamide in a mouse model of mastocytoma. In addition, the antitumour effects of cyclophosphamide were reduced in germ-free mice compared with mice that had a normal gut microbiota.

Corresponding author Laurence Zitvogel notes that it is now important to replicate these findings in patients. It might then be possible to treat patients with probiotics before they undergo chemotherapy to enable 'good bacteria' to penetrate the gut epithelial barrier.

In the second study, cells from three different cancer cell lines were transplanted under the skin of mice at a site distant from the gut microbiota. Once the tumours had grown to a diameter of $>5 \mathrm{~mm}$, the mice underwent immunotherapy and chemotherapy with either oxaliplatin or cisplatin.

They found that the tumours of germfree mice and mice treated with antibiotics did not respond to the therapy as well as control mice. In these mice, tumourinfiltrating myeloid-derived cells produced reduced levels of several cytokines, including TNF, after treatment. Several Gram-positive and Gram-negative species of bacteria primed tumour myeloid cells for the response to immunotherapy in mice with an intact gut microbiota. By contrast, several Lactobacillus species limited the response in mice with dysbiosis. Unexpectedly, the myeloid-derived cells of these mice also produced lower levels of reactive oxygen species than mice with an intact gut microbiota, which was partly responsible for the decreased effectiveness of oxaliplatin. "Thus, an intact commensal microbiota modulates myeloidderived cell functions in the tumour microenvironment and it is required for the early cytotoxic response to cancer immunotherapy and chemotherapy," says author Giorgio Trinchieri.

"These data not only represent a paradigm shift in our understanding of the mechanism of action of anti-cancer drugs but also have potential clinical relevance by underscoring the risk associated with antibiotic medication during cancer treatment and by suggesting the possibility to alter the gut microbiota to improve anti-cancer drug efficacy," explains Trinchieri.

The findings of these two papers suggest that use of antibiotics over a patient's lifetime, or to treat infection or adverse events during cancer treatments, could affect the efficacy of chemotherapy. Thus, Trinchieri and Romina Goldszmid, the two lead authors of the paper, are planning to elucidate the molecular signalling involved in the gut microbiota's effect on treatment response in mouse models. The researchers also plan to design clinical studies involving giving antibiotics to healthy volunteers to investigate the effect of antibiotics on the molecular mechanisms regulating inflammation.

"It is exciting to think that a 'personalized' medicine could be applied based on microbiome identity," says Christian Jobin (University of Florida), who was not involved in either study. "For instance, one could imagine that, in the future, we could determine the degree of cancer drug responsiveness based on microbiome composition."

\section{Claire Greenhill}

Original articles Viaud, S. et al. The intestinal microbiota modulates the anticancer effects of cyclophosphamide. Science 342, 971-976 (2013) | lida, N. et al. Commensal bacteria control cancer response to therapy by modulating the tumor microenvironment. Science 342, 967-970 (2013) 